\title{
Ferritas Ni-Zn: breve revisão sobre o processo convencional de fabricação e as propriedades permeabilidade magnética e constante dielétrica
}

\section{(Ni-Zn ferrites: brief review on the conventional method of fabrication and the magnetic permeability and dielectric constant properties)}

\author{
V. L. O. Brito \\ Instituto de Estudos Avançados - Divisão de Física Aplicada \\ Rodovia dos Tamoios, km 5,5, Torrão de Ouro, S. José dos Campos, SP \\ vlobrito@ieav.cta.br
}

\begin{abstract}
Resumo
As ferritas Ni-Zn são cerâmicas magnéticas que têm sido amplamente aplicadas na fabricação de componentes elétricos e eletrônicos. Esses materiais têm sido estudados há décadas por diversos autores, os quais têm definido a relação entre as propriedades magnéticas e elétricas dessas ferritas em função de diversos fatores, tais como as variáveis do processo de fabricação, composição química e microestrutura. Este trabalho mostra uma revisão dos principais fatores que influenciam as propriedades das ferritas Ni-Zn, com foco na permeabilidade magnética e constante dielétrica e enfatizando as características do método cerâmico convencional na fabricação desses materiais.

Palavras-chave: ferritas, cerâmicas magnéticas, permeabilidade magnética, constante dielétrica.
\end{abstract}

Abstract

$\mathrm{Ni}$-Zn ferrites are magnetic ceramics that have been widely used on the fabrication of electrical and electronic components. Such materials have been studied for many years by several researchers and their magnetic and electrical properties have been assessed in relation to many factors, such as chemical composition, fabrication methods, and microstructure. This work makes a review of the main factors influencing the properties of $\mathrm{Ni}$-Zn ferrites. It is emphasized the magnetic permeability and dielectric constant, as well as the characteristics of the conventional ceramic method on the fabrication of these ferrites.

Keywords: ferrites, magnetic ceramics, magnetic permeability, dielectric constant.

\section{INTRODUÇÃO}

A Divisão de Física Aplicada do Instituto de Estudos Avançados(IEAv/EFA) adquiriu, na década de 80, experiência na fabricação de ferritas para a confecção de componentes do acelerador linear de elétrons sendo construído por essa Divisão [1]. Ainda nessa linha de pesquisa, em 2003 foi iniciado um projeto sobre a aplicação de ferritas $\mathrm{Ni}$ Zn na fabricação de dispositivos eletromagnéticos e uma pesquisa bibliográfica contínua sobre estes materiais tem sido feita desde então. $\mathrm{O}$ foco da pesquisa em andamento é as propriedades permeabilidade magnética e constante dielétrica de ferritas Ni-Zn fabricadas pelo método cerâmico convencional. Tendo em vista tal foco, este trabalho tem como objetivo mostrar uma breve revisão bibliográfica sobre os aspectos mais relevantes sobre o tema.

As ferritas Ni-Zn vêm sendo estudadas de forma bastante detalhada nas últimas décadas e muito tem sido publicado a respeito destes materiais: suas propriedades têm sido caracterizadas em função de diversos fatores e melhoradas com a aplicação das novas tecnologias que vêm surgindo. As ferritas Ni-Zn possuem permeabilidade magnética relativamente alta em freqüências elevadas. A literatura mostra que a permeabilidade magnética das ferritas em geral apresenta um valor aproximadamente constante até uma determinada freqüência, acima da qual esta propriedade diminui drasticamente. No caso de ferritas Ni-Zn esta freqüência está por volta de 1-10 MHz [2-4]. Outras ferritas de alta permeabilidade magnética, tais como as do tipo Mn$\mathrm{Zn}$, têm a permeabilidade magnética drasticamente afetada pela quantidade de oxigênio incorporada da atmosfera de sinterização durante o resfriamento [5], tornando necessário um rigoroso controle da pressão parcial do oxigênio na atmosfera de sinterização. A ferrita Ni-Zn, por sua vez, é menos afetada pela atmosfera de sinterização e pode ser sinterizada ao ar, o que resulta em um processo de fabricação 
mais simplificado. As propriedades magnéticas e elétricas das ferritas Ni-Zn permitem um amplo campo de aplicações deste tipo de material em componentes elétricos e eletrônicos, tais como em núcleos de transformadores, cabeçotes de gravação de fitas magnéticas, isoladores, circuladores e outros dispositivos que operam em alta freqüência. A literatura também aponta a possibilidade da aplicação deste tipo de ferrita como transdutores magnetoelásticos para a medição de forças e de tensões de compressão [6].

\section{CONSIDERAÇÕES GERAIS SOBRE FERRITAS Ni-Zn}

\section{Estrutura cristalina}

As ferritas Ni-Zn possuem estrutura cristalina do tipo espinélio, que é uma estrutura cúbica, formada por oito unidades do tipo $\mathrm{AB}_{2} \mathrm{O}_{4}$. $\mathbf{A}$ corresponde a um cátion divalente nos interstícios tetraédricos e $\mathbf{B}$ a um cátion trivalente nos interstícios octaédricos da estrutura cúbica de face centrada formada pelos átomos de oxigênio. Dependendo da posição dos cátions na rede cristalina, a estrutura espinélio é definida [7] como normal (no caso de $\left(\mathrm{Me}^{2+}\right)\left[\mathrm{Fe}^{3+}\right] \mathrm{O}_{4}$, sendo Me um metal) e inversa (no caso de $\left(\mathrm{Fe}^{3+}\right)\left[\mathrm{Me}^{2+} \mathrm{Fe}^{3+}\right] \mathrm{O}_{4}$.

Quando se adicionam íons $\mathrm{Zn}^{2+}$ à estrutura de uma ferrita de níquel, esses íons deslocarão cátions $\mathrm{Fe}^{3+}$ da posição $\mathbf{A}$ para a posição $\mathbf{B}$. Sendo assim, nas ferritas Ni-Zn os íons de ferro ocuparão tanto as posições $\mathbf{A}$ quanto as posições $\mathbf{B}$, sendo a distribuição desses íons em tais posições dependente da proporção Ni/Zn da ferrita [8]. A célula unitária espinélio de uma ferrita do tipo Ni-Zn pode ser então considerada [9] como sendo formada de oito unidades do tipo:

$$
\left(\mathrm{Zn}_{\mathrm{x}}^{2+} \mathrm{Fe}_{1-\mathrm{x}}^{3+}\right)\left[\mathrm{Ni}_{1-\mathrm{x}}^{2+} \mathrm{Fe}_{1+\mathrm{x}}^{3+}\right] \mathrm{O}_{4}^{2-}
$$

A literatura [9-10] mostra que o parâmetro de rede das ferritas Ni-Zn aumenta linearmente com o aumento do teor

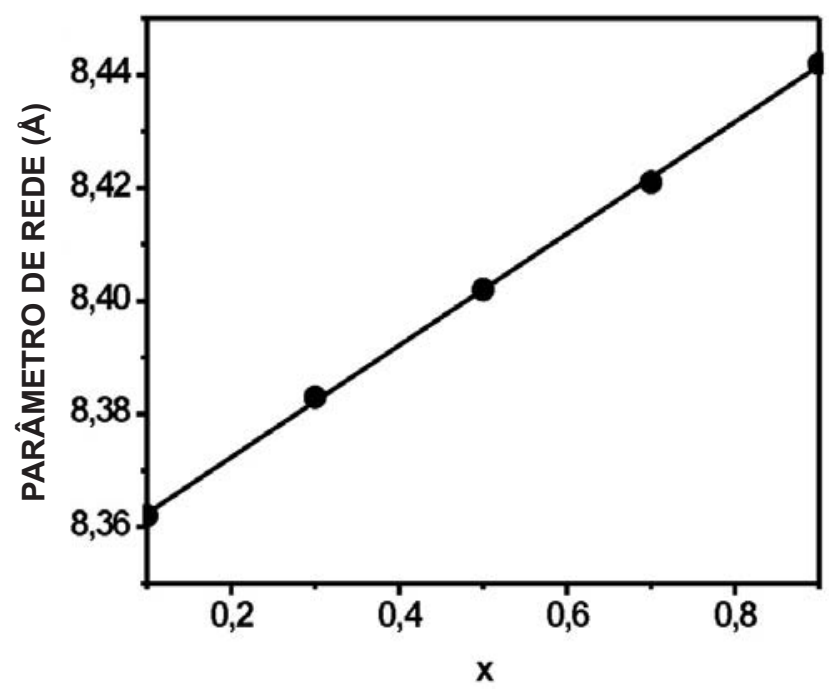

Figura 1: Parâmetro de rede em função da composição de uma ferrita $\mathrm{Ni}_{1-\mathrm{x}} \mathrm{Zn}_{\mathrm{x}} \mathrm{Fe}_{2} \mathrm{O}_{4}[9]$.

[Figure 1: Compositional variation of the lattice constant for a $\mathrm{Ni} \mathrm{Zn}_{\mathrm{I}} \mathrm{Fe}_{2} \mathrm{O}_{4}$ ferrite [9].] de zinco e esse aumento ocorre pois o raio do íon $\mathrm{Zn}^{2+}$ é cerca de 13,5\% maior do que o raio do íon $\mathrm{Ni}^{2+}$. A Fig. 1, construída com dados da literatura [9], mostra a variação do parâmetro de rede com a composição de uma ferrita Ni-Zn.

Processos de fabricação: o método cerâmico convencional e os métodos químicos

Vários métodos químicos para a síntese de ferritas têm sido desenvolvidos, tornando-se possível a obtenção, em laboratório, de partículas nanométricas de tamanho uniforme e de alta pureza. Pode-se citar, por exemplo, um trabalho recentemente publicado, relatando a obtenção de partículas nanométricas de ferritas Ni-Zn por meio do método de combustão induzida por microondas [11]. Foi também recentemente relatada a obtenção de uma ferrita $\mathrm{Ni}-\mathrm{Zn}$ por moagem de alta energia de uma mistura $\mathrm{Fe}_{3} \mathrm{O}_{4}+\mathrm{NiO}+\mathrm{ZnO}$ [12], chegando-se a um pó com granulometria entre $10-50 \mathrm{~nm}$.

$\mathrm{O}$ uso de pós nanométricos obtidos por método químico tem apresentado diversas vantagens, tais como a possibilidade de obtenção de densidades relativas muito elevadas, chegando a 98\% [13]. Com o avanço da nanotecnologia, atualmente é possível não apenas obter partículas nanométricas de ferritas mas também controlar o formato de tais partículas. Já foi relatada [14] a sintetização de uma ferrita $\mathrm{Ni}_{0,5} \mathrm{Zn}_{0,5} \mathrm{Fe}_{2} \mathrm{O}_{3}$ na forma de agulhas com alta razão de aspecto e também de partículas perfeitamente esféricas de ferrita $\mathrm{Ni}-\mathrm{Zn}$ em uma matriz de sílica [15].

Embora os métodos químicos produzam materiais de alta qualidade, com o método cerâmico convencional se consegue processar com maior facilidade a matéria-prima em grande escala, razão pela qual este tem sido o método mais aplicado nas indústrias.

\section{O MÉTODO CERÂMICO CONVENCIONAL NA FABRICAÇÃO DE FERRITAS}

As principais etapas do processamento de ferritas pelo método cerâmico convencional são comentadas a seguir.

\section{Formulação da composição}

Nesta etapa, as matérias-primas (na maioria das vezes, óxidos) são pesadas na proporção adequada. Para ferritas Ni-Zn normalmente se parte dos óxidos $\mathrm{NiO}, \mathrm{ZnO}$ e $\mathrm{Fe}_{2} \mathrm{O}_{3}$. Na seleção da matéria prima, é ressaltada a importância da avaliação dos seguintes fatores [16]: nível de impurezas, reatividade (tamanho de partícula), processamento utilizado no pó, e custo.

$\mathrm{O}$ tamanho da partícula do óxido de ferro $\left(\mathrm{Fe}_{2} \mathrm{O}_{3}\right)$ utilizado como matéria-prima pode afetar as propriedades finais da ferrita $\mathrm{Ni}-\mathrm{Zn}$ [17]. Foi verificado que quando se aumenta o tamanho da partícula do $\mathrm{Fe}_{2} \mathrm{O}_{3}$, a resistividade da ferrita Ni-Zn aumenta e as perdas e o tamanho de grão diminuem após a sinterização. No trabalho citado, variou-se o tamanho médio de partícula do $\mathrm{Fe}_{2} \mathrm{O}_{3}$ de $0,18 \mu \mathrm{m}$ a $0,53 \mu \mathrm{m}$, sendo acima $0,28 \mu \mathrm{m}$ encontradas as menores perdas, menores tamanhos de grão e maior resistividade. 


\section{Moagem/mistura da matéria prima}

As matérias-primas, pesadas nas proporções adequadas, são misturadas com o objetivo de eliminar aglomerados de partículas e/ou reduzir o tamanho de partícula. Este processo facilita a calcinação ou sinterização posteriores. Em escala industrial, esta moagem pode ser feita de forma contínua.

A moagem a úmido é mais rápida do que a moagem a seco e facilita a separação entre a carga e o meio de moagem. O líquido deve ser adicionado em volume suficiente para formar uma carga com boa fluidez. Os moinhos mais utilizados são os do tipo atritor, por jato, vibratório e de bolas.

Os corpos moedores, normalmente de aço, aumentam a quantidade de ferro no material moído em cerca de $1 \%$ [18]. Essa quantidade de ferro adicional que é introduzida durante o processo deve ser levada em consideração quando se define a estequiometria da ferrita, já que se sabe [19] que esse é um fator que afeta a permeabilidade magnética das ferritas. Já foi relatado que a deficiência em ferro, mesmo pequena, facilita a formação da ferrita [20].

Para facilitar a transferência da matéria-prima para a próxima etapa do processo, deve-se executar a operação de pelotização, a qual pode ser feita por compactação, extrusão ou em pratos pelotizadores [18].

\section{Pré-sinterização}

A pré-sinterização é um tratamento térmico anterior à sinterização que tem como objetivo causar a interação entre os constituintes por interdifusão dos seus íons e, assim, reduzir a extensão necessária da difusão a ocorrer durante a sinterização [21]. O tratamento normalmente é feito com temperaturas entre $700-1000{ }^{\circ} \mathrm{C}$ ao ar. Nas ferritas $\mathrm{Ni}-\mathrm{Zn}$ chega-se a atingir quase $100 \%$ de conversão da estrutura cristalina em espinélio na pré-sinterização [7]. O tamanho de partícula do pó também influi nesse percentual de conversão [17] pois o pó constituído de partículas menores é mais reativo. A pré-sinterização é um dos fatores determinantes das propriedades finais da ferrita, pois ela afetará a reatividade do pó que constituirá o compacto a ser sinterizado, bem como a sua contração após a sinterização.

\section{Moagem para homogeneização e ajuste da reatividade}

O material pré-sinterizado deverá ser novamente moído/misturado para homogeneização da composição e uniformização do tamanho de partícula do pó. Esta moagem não deve se estender demais, pois isto gerará uma certa quantidade de partículas ultrafinas que poderão causar crescimento descontínuo dos grãos durante a sinterização. Por esse motivo afirma-se que, na etapa de moagem, o controle da largura da banda de distribuição do tamanho das partículas é mais importante que o controle do tamanho médio das partículas [22]. O uso de pós ultrafinos é interessante quando se deseja uma microestrutura formada de grãos pequenos. No entanto, quando se utiliza pós com partículas menores que $0,1 \mu \mathrm{m}$ problemas de crescimento descontínuo de grão são freqüentes [23].

O material calcinado possui dureza mais elevada e por isso deve-se escolher apropriadamente o material do meio de moagem, de modo a evitar abrasão do mesmo e contaminação do material moído. Assim como na etapa de mistura/moagem da matéria-prima, o material normalmente usado nos corpos moedores é o aço de baixo carbono ou ao cromo [18].

Nesta etapa é aconselhável executar uma análise química do material para que se possam fazer eventuais correções da composição. Nesta última moagem é muito comum se utilizar aditivos tais como dispersantes, aglutinantes e lubrificantes que vão facilitar, respectivamente, a dispersão da matériaprima durante a moagem, a aglutinação das partículas na secagem por spray e o aumento da resistência do compacto durante a prensagem.

Caso a moagem tenha sido realizada a úmido, o material deverá ser seco. O processo de secagem por spray é uma das soluções para secagem utilizadas na indústria. Neste processo, a solução contendo as partículas do pó é pulverizada e o líquido é evaporado por um jato de ar quente. Os aglomerados muito finos e mais leves são separados. Os aglomerados mais pesados serão aproveitados e deverão conferir uma boa fluidez ao material, permitido que o mesmo preencha com maior facilidade o molde de prensagem.

A moagem a seco pode ser uma alternativa à moagem a úmido no processo industrial. Se feito por processo contínuo, esse tipo de moagem é bastante adequado para o processamento em larga escala [18]. No entanto, foi mostrado em laboratório que o método à úmido produz um pó mais reativo, resultando na necessidade de menores temperaturas de sinterização e em uma microestrutura mais uniforme nas ferritas Ni-Zn [24].

O uso da técnica de carregamento posterior ("postcharging") [25] foi proposto para o controle da microestrutura das ferritas. De acordo com esta técnica, os óxidos com menor volume específico que a ferrita e cuja difusão na ferrita seja mais rápida que a da ferrita neles, devem ser adicionados parte na mistura inicial e parte no pó pré-sinterizado. Essa técnica diminui a porosidade e promove a contenção do crescimento de grão durante a sinterização.

\section{Formação das peças}

No planejamento da etapa de formação das peças para sinterização, devem ser levadas em consideração as possíveis deformações e a contração, que resultam do processo de sinterização. Nesse planejamento, um modelo numérico tais como alguns mostrados [26] para a deformação da ferrita durante a sinterização, pode ser bastante útil.

A peça final poderá ser formada por vários processos, tais como prensagem, extrusão e injeção. Um dos processos mais simples é o por prensagem uniaxial. Um outro método de prensagem bastante eficiente é o método isostático, no qual a amostra é colocada em um molde flexível sujeito à pressão externa de um líquido ou de um gás. 
Para se conseguir densidades ainda mais elevadas, pode-se utilizar prensagem isostática a quente. Oberbeck [27] mostra um método industrial de formação, utilizando prensagem a quente em uma das etapas, capaz de gerar peças com porosidade bastante reduzida.

A prensagem de pós ultrafinos pode apresentar problemas operacionais tais como desgaste excessivo da matriz, emperramento e má fluidez / mau empacotamento do pó. Para minimizar estes problemas, quando se trabalha com partículas nanométricas, é altamente aconselhável que as mesmas sejam aglomeradas em partículas maiores com o auxílio de aditivos [28].

\section{Sinterização}

A temperatura e o tempo de sinterização são dois parâmetros cujos valores normalmente são estabelecidos de forma inversamente proporcional, sendo recomendado favorecer o parâmetro tempo em detrimento da temperatura. Temperaturas muito altas podem causar a decomposição da estrutura espinélio, criando gradientes de composição na ferrita e aumentando a porosidade.

O tempo deverá ser determinado de forma que, na temperatura definida para a sinterização, possam ocorrer todas as reações no estado sólido para a formação da estrutura espinélio sem, no entanto, ocasionar crescimento descontínuo de grão. Heck [29], em sua revisão bibliográfica, mostra que para uma dada ferrita $\mathrm{Ni}-\mathrm{Zn}$ os maiores valores de permeabilidade magnética foram obtidos para tempos mais longos de sinterização. Para a composição apresentada pelo autor, o valor mais alto de permeabilidade atingido foi de um pouco menos de 5.000 , a partir de uma sinterização por 20 horas em uma temperatura entre $1200-1250^{\circ} \mathrm{C}$.

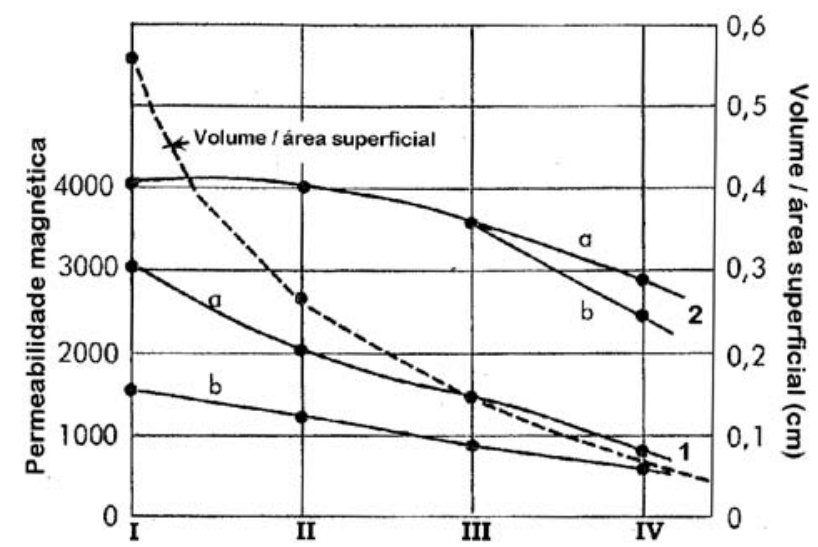

Figura 2: Resultados de permeabilidade magnética para amostras de ferritas de diferentes dimensões [30]. (1) Aquecida por $3 \mathrm{~h}$ ao ar a $1380{ }^{\circ} \mathrm{C}$ e resfriada rapidamente a partir de $600{ }^{\circ} \mathrm{C}$. (2) Aquecida de $1240{ }^{\circ} \mathrm{C}$ a $1380{ }^{\circ} \mathrm{C}$ em $3 \mathrm{~h}$ e em atmosfera cuidadosamente controlada. (a) Pré-sinterizada. (b) Sem pré-sinterização.

[Figure 2: Magnetic permeability measurements from ferrite samples of different dimensions [30]. (1) Heated at $1380^{\circ} \mathrm{C}$ for $3 \mathrm{~h}$ in air and quenched from $600^{\circ} \mathrm{C}$. (2) Heated from $1240^{\circ} \mathrm{C}$ to $1380^{\circ} \mathrm{C}$ in $3 \mathrm{~h}$, under controlled atmosphere. (a) Calcined. (b) Not calcined.]
Tabela I - Dimensões em mm dos toróides mencionados na Fig. 2.

[Table I - Dimensions (in $\mathrm{mm}$ ) of the toroids mentioned on Fig. 2.]

\begin{tabular}{ccccc}
\hline & I & II & III & IV \\
\hline $\mathrm{D}_{\mathrm{e}}$ & 100 & 40 & 30 & 23 \\
$\mathrm{D}_{\mathrm{i}}$ & 18 & 18 & 18 & 18 \\
$\mathrm{~h}$ & 15 & 10 & 6 & 15 \\
\hline
\end{tabular}

As dimensões da amostra influenciarão diretamente a velocidade de aquecimento e de resfriamento do material e, dessa forma, afetarão a estrutura final da ferrita. A Fig. 2 [30] mostra resultados de permeabilidade magnética, em uma dada ferrita, para medições feitas em quatro toróides (I a IV) variando o diâmetro externo $\mathrm{D}_{\mathrm{e}}$, o diâmetro interno $\mathrm{D}_{\mathrm{i}}$ e a altura $\mathrm{h}$ mostrados na Tabela I.

Observando-se a Fig. 1 conclui-se que os seguintes fatores contribuíram para a obtenção de valores mais elevados de permeabilidade magnética: maior relação volume/área superficial, execução da etapa de pré-sinterização, e controle da atmosfera de sinterização.

Embora as ferritas Ni-Zn sejam menos afetadas pela atmosfera de sinterização, um controle da pressão parcial de oxigênio da atmosfera de sinterização pode ser útil. Esse controle minimiza o gradiente de composição que pode ocorrer na seção transversal da amostra, devido à evaporação de elementos oriundos da decomposição da estrutura espinélio em altas temperaturas. Para evitar a dissociação do $\mathrm{ZnO}$ na sinterização de ferritas à base desse óxido, deve-se manter uma pequena pressão de oxigênio na atmosfera de sinterização.

Blank [31] propôs um diagrama universal de atmosfera de equilíbrio para sinterização de ferritas espinélio. Com base nas linhas desse diagrama, pode-se ajustar o percentual adequado de oxigênio da atmosfera de sinterização em função da temperatura de sinterização.

Em um estudo do efeito da atmosfera da sinterização a $1180{ }^{\circ} \mathrm{C}$ de ferritas $\mathrm{Ni}-\mathrm{Zn}$ [32], variando a pressão parcial de oxigênio $\mathrm{p}\left(\mathrm{O}_{2}\right)$ de 0 a $100 \%$ em uma atmosfera constituída de uma mistura de $\mathrm{N}_{2}+\mathrm{O}_{2}$, foram verificados os seguintes efeitos nas ferritas estudadas: aumento da permeabilidade magnética com o aumento de $\mathrm{p}\left(\mathrm{O}_{2}\right)$, sendo $20 \%$ a pressão parcial com a qual foram observados os menores coeficientes de perdas a $100 \mathrm{kHz}$, precipitação de $\mathrm{ZnO}$ nos contornos de grão para $\mathrm{p}\left(\mathrm{O}_{2}\right)<20 \%$.

A mesma ferrita, sinterizada a uma temperatura mais baixa $\left(1150{ }^{\circ} \mathrm{C}\right)$, não apresentou precipitados de $\mathrm{ZnO}$ quando sinterizada ao ar [33].

Existem na literatura alguns trabalhos a respeito de sinterização de ferritas por meio de microondas [34-36]. A literatura aponta como principal vantagem deste método o ciclo mais curto de sinterização, com maior eficiência energética. Foi verificado [35] que as ferritas Ni-Zn sinterizadas por meio de microondas apresentaram constante 
dielétrica mais baixa que as ferritas sinterizadas pelo método convencional. Os valores obtidos nesse trabalho serão mostrados em uma próxima seção.

Outro método menos convencional também já estudado para a sinterização de ferritas [37] é o método "sparkplasma", no qual o pó é sinterizado sob pressão uniaxial e aquecido por efeito Joule e pelo efeito do plasma gerado entre as partículas do pó. O método, que requer um tempo de sinterização mais curto e temperaturas mais baixas, gera um material menos poroso e facilita a obtenção de uma microestrutura com grãos pequenos.

\section{INFLUÊNCIA DA COMPOSIÇÃO QUÍMICA NAS PROPRIEDADES DAS FERRITAS Ni-Zn}

A literatura sobre a influência da composição química nas propriedades das ferritas $\mathrm{Ni}-\mathrm{Zn}$ é bastante vasta. A seguir são mostrados resultados de alguns autores para permeabilidade magnética e constante dielétrica desses materiais, variando-se a estequiometria da ferrita e testandose o uso de dopantes.

Influência da proporção $\mathrm{Ni} / \mathrm{Zn}$

\section{Influência na permeabilidade magnética}

Heck [29] elaborou uma revisão bibliográfica abrangendo a variação da permeabilidade magnética de ferritas Ni-Zn, fabricadas pelo método cerâmico convencional, em função da proporção dos óxidos $\mathrm{NiO}, \mathrm{ZnO}$ e $\mathrm{Fe}_{2} \mathrm{O}_{3}$ utilizada na fabricação. $\mathrm{O}$ autor mostra que teor de $50 \% \mathrm{Fe}_{2} \mathrm{O}_{3}$ é o teor no qual é possível obter maior permeabilidade magnética ( $\left.\mu^{\prime}\right)$ e maior variação desta propriedade alterando-se a proporção $\mathrm{NiO} / \mathrm{ZnO}$ na formulação. A Fig. 2 sumariza os resultados apresentados no trabalho.

A Tabela II mostra os valores de permeabilidade magnética encontrados na literatura para ferritas Ni-Zn de diversas estequiometrias. Verifica-se na tabela que os valores mais altos de permeabilidade foram os da ferrita $\mathrm{Ni}_{0,3} \mathrm{Zn}_{0,7} \mathrm{Fe}_{2} \mathrm{O}_{4}$, tanto em baixas quanto em altas freqüências. Já os valores mais baixos de permeabilidade foram os da ferrita $\mathrm{Ni}_{0,8} \mathrm{Zn}_{0,2} \mathrm{Fe}_{2} \mathrm{O}_{4}$.

Como esperado, pode-se também observar que os valores de permeabilidade magnética são bastante afetados pelo tipo de processo de fabricação e pelos seus parâmetros. As variações de valores entre ferritas de mesma composição estão provavelmente relacionadas às diferenças microestruturais resultantes de variações no processo de fabricação.

\section{Influência na constante dielétrica}

Os valores de constante dielétrica $\left(\varepsilon^{\prime}\right)$ e de perdas dielétricas $(\operatorname{tg} \delta)$ das ferritas Ni-Zn variam bastante com a freqüência e $\varepsilon$ ' tende a ser bem alto em freqüências baixas, caindo bruscamente a partir de cerca $10 \mathrm{kHz}$. A Tabela III mostra alguns resultados de $\varepsilon$ ' pesquisados da literatura para diversos tipos ferritas $\mathrm{Ni}-\mathrm{Zn}$ em determinadas freqüências.

Um artigo [44] mostrou o estudo da variação das propriedades dielétricas das ferritas $\mathrm{Ni}_{1-\mathrm{x}} \mathrm{Zn}_{\mathrm{x}} \mathrm{Fe}_{2} \mathrm{O}_{3}$, de 100 $\mathrm{kHz}$ a $1 \mathrm{MHz}$, com x variando de 0,2 a 1,0. Foi observada uma

Tabela II - Permeabilidade magnética ( $\mu$ ') de algumas ferritas Ni-Zn.

[Table II - Magnetic permeability ( $\mu$ ') of some Ni-Zn ferrites.]

\begin{tabular}{|c|c|c|c|c|c|}
\hline Composição & Fabricação & Sinterização & $\mu^{\prime}$ & Freqüência & Ref. \\
\hline $\mathrm{Ni}_{024} \mathrm{Zn}_{065} \mathrm{Fe}_{204} \mathrm{O}_{4}$ & Não informado & Não informado & 1400 & $100 \mathrm{kHz}$ & 3 \\
\hline $\mathrm{Ni}_{03}^{0,24} \mathrm{Zn}_{07}^{0,63} \mathrm{Fe}_{2} \mathrm{O}_{4}{ }^{2,04}$ & Convencional & $1150{ }^{\circ} \mathrm{C}-96 \mathrm{~h}$ & 3422 & $10 \mathrm{kHz}$ & 38 \\
\hline $\mathrm{Ni}_{0,3}^{0,3} \mathrm{Zn}_{0,7}^{0,7} \mathrm{Fe}_{2}^{2} \mathrm{O}_{4}^{4}$ & Convencional & $1170{ }^{\circ} \mathrm{C}-20 \mathrm{~h}$ & 3360 & $10 \mathrm{kHz}$ & 39 \\
\hline $\mathrm{Ni}_{0,3}^{0,3} \mathrm{Zn}_{0,7} \mathrm{Fe}_{2} \mathrm{O}_{4}$ & $\begin{array}{l}\text { Convencional com } \\
\text { prensagem a quente }\end{array}$ & $950{ }^{\circ} \mathrm{C}-1 \mathrm{~h}$ & 835 & $10 \mathrm{kHz}$ & 39 \\
\hline $\mathrm{Ni}_{0,3} \mathrm{Zn}_{0,7} \mathrm{Fe}_{2} \mathrm{O}_{4}$ & Convencional & $1100^{\circ} \mathrm{C}-6 \mathrm{~h}$ & $250 *$ & $1 \mathrm{MHz}$ & 42 \\
\hline $\mathrm{Ni}_{0,35}{ }^{0,3} \mathrm{Zn}_{0,65} \mathrm{Fe}_{2} \mathrm{O}_{4}$ & Convencional & $1150{ }^{\circ} \mathrm{C}-96 \mathrm{~h}$ & 1202 & $10 \mathrm{kHz}$ & 38 \\
\hline $\mathrm{Ni}_{0,4}^{0,35} \mathrm{Zn}_{0,6} \mathrm{Fe}_{2} \mathrm{O}_{4}$ & Convencional & $1150{ }^{\circ} \mathrm{C}-96 \mathrm{~h}$ & 1665 & $10 \mathrm{kHz}$ & 38 \\
\hline $\mathrm{Ni}_{0,4}^{0,4} \mathrm{Zn}_{0,6}^{0,6} \mathrm{Fe}_{2}^{2} \mathrm{O}_{4}^{4}$ & Convencional & $1170{ }^{\circ} \mathrm{C}-20 \mathrm{~h}$ & 990 & $10 \mathrm{kHz}$ & 39 \\
\hline $\mathrm{Ni}_{0,4}^{0,4} \mathrm{Zn}_{0,6}^{0,6} \mathrm{Fe}_{2} \mathrm{O}_{4}$ & $\begin{array}{l}\text { Convencional com } \\
\text { prensagem a quente }\end{array}$ & $950^{\circ} \mathrm{C}-1 \mathrm{~h}$ & 555 & $10 \mathrm{kHz}$ & 39 \\
\hline $\mathrm{Ni}_{04} \mathrm{Zn}_{06} \mathrm{Fe}_{2} \mathrm{O}_{4}$ & Convencional & $1100^{\circ} \mathrm{C}-6 \mathrm{~h}$ & $187^{*}$ & $1 \mathrm{MHz}$ & 42 \\
\hline $\mathrm{Ni}_{0,5} \mathrm{Zn}_{0,5} \mathrm{Fe}_{2} \mathrm{O}_{4}$ & Convencional & $1200^{\circ} \mathrm{C}-4 \mathrm{~h}$ & 304 & $1 \mathrm{kHz}$ & 40 \\
\hline $\mathrm{Ni}_{0,5} \mathrm{Zn}_{0,5} \mathrm{Fe}_{2} \mathrm{O}_{4}$ & Convencional & $1100^{\circ} \mathrm{C}-6 \mathrm{~h}$ & $138^{*}$ & $1 \mathrm{MHz}$ & 42 \\
\hline $\mathrm{Ni}_{0,65} \mathrm{Zn}_{0,35} \mathrm{Fe}_{2} \mathrm{O}_{4}$ & Convencional & $1300^{\circ} \mathrm{C}-4 \mathrm{~h}$ & 415 & $10 \mathrm{kHz}$ & 41 \\
\hline $\mathrm{Ni}_{0,7} \mathrm{Zn}_{0,3} \mathrm{Fe}_{2} \mathrm{O}_{4}$ & Convencional & $1300^{\circ} \mathrm{C}-4 \mathrm{~h}$ & $185^{*}$ & $100 \mathrm{kHz}$ & 4 \\
\hline $\mathrm{Ni}_{0,8}^{0,} \mathrm{Zn}_{0,2} \mathrm{Fe}_{2} \mathrm{O}_{4}$ & Síntese por combustão & $1350^{\circ} \mathrm{C}-3 \mathrm{~h}$ & $47^{*}$ & $1 \mathrm{kHz}$ & 43 \\
\hline $\mathrm{Ni}_{0,8}^{0,8} \mathrm{Zn}_{0,2} \mathrm{Fe}_{2} \mathrm{O}_{4}^{4}$ & Síntese por combustão & $1350^{\circ} \mathrm{C}-3 \mathrm{~h}$ & $21^{*}$ & $10 \mathrm{kHz}$ & 43 \\
\hline $\mathrm{Ni}_{0,8}^{, 6} \mathrm{Zn}_{0,2} \mathrm{Fe}_{2} \mathrm{O}_{4}$ & Convencional & $1350{ }^{\circ} \mathrm{C}-3 \mathrm{~h}$ & $25^{*}$ & $1 \mathrm{kHz}$ & 43 \\
\hline $\mathrm{Ni}_{0,8}^{0} \mathrm{Zn}_{0,2} \mathrm{Fe}_{2} \mathrm{O}_{4}$ & Convencional & $1350{ }^{\circ} \mathrm{C}-3 \mathrm{~h}$ & $13^{*}$ & $10 \mathrm{kHz}$ & 43 \\
\hline
\end{tabular}


Tabela III - Constantes dielétricas ( $\left.\varepsilon^{\prime}\right)$ de algumas ferritas Ni-Zn. [Table III - Dielectric constants ( $\left.\varepsilon^{\prime}\right)$ of some Ni-Zn ferrites.]

\begin{tabular}{|c|c|c|c|c|c|}
\hline Composição & Fabricação & Sinterização & $\varepsilon$ & Freqüência & Ref. \\
\hline $\mathrm{Ni}_{02} \mathrm{Zn}_{08} \mathrm{Fe}_{2} \mathrm{O}_{4}$ & Convencional & $1200^{\circ} \mathrm{C}-6 \mathrm{~h}$ & 53,59 & $100 \mathrm{kHz}$ & 44 \\
\hline $\mathrm{Ni}_{0,2} \mathrm{Zn}_{0} \mathrm{Fe}_{2} \mathrm{O}_{4}^{4}$ & Convencional & $1275^{\circ} \mathrm{C}-0,5 \mathrm{~h}$ & 1155 & $1 \mathrm{kHz}$ & 35 \\
\hline $\mathrm{Ni}_{0,2}^{0,2} \mathrm{Zn}_{08} \mathrm{Fe}_{2} \mathrm{O}_{4}^{4}$ & Convencional & Microondas $1275^{\circ} \mathrm{C}-0,5 \mathrm{~h}$ & 22,80 & $1 \mathrm{kHz}$ & 35 \\
\hline $\mathrm{Ni}_{04} \mathrm{Zn}_{06} \mathrm{Fe}_{2} \mathrm{O}_{4}$ & Convencional & $1200{ }^{\circ} \mathrm{C}-6 \mathrm{~h}$ & 45,73 & $100 \mathrm{kHz}$ & 44 \\
\hline $\mathrm{Ni}^{2} \mathrm{Zn}_{06} \mathrm{Fe}_{2} \mathrm{O}_{4}$ & Convencional & $1275^{\circ} \mathrm{C}-0,5 \mathrm{~h}$ & 396 & $1 \mathrm{kHz}$ & 35 \\
\hline $\mathrm{Ni}_{04}^{0,4} \mathrm{Zn}_{06} \mathrm{Fe}_{2} \mathrm{O}_{4}^{4}$ & Convencional & Microondas $1275^{\circ} \mathrm{C}-0,5 \mathrm{~h}$ & 7,57 & $1 \mathrm{kHz}$ & 35 \\
\hline $\mathrm{Ni}_{04} \mathrm{Zn}_{06} \mathrm{Fe}_{2} \mathrm{O}_{4}$ & Precursor de citrato & $1200{ }^{\circ} \mathrm{C}-1 \mathrm{~h}$ & $30^{*}$ & $30 \mathrm{~Hz}$ & 45 \\
\hline $\mathrm{Ni}_{05} \mathrm{Zn}_{0} \mathrm{Fe}_{2} \mathrm{O}_{4}^{4}$ & Convencional & $1200^{\circ} \mathrm{C}-6 \mathrm{~h}$ & 44,22 & $100 \mathrm{kHz}$ & 44 \\
\hline $\mathrm{Ni}_{0,5} \mathrm{Zn}_{0,5} \mathrm{Fe}_{2} \mathrm{O}_{4}$ & Precursor de citrato & $1200{ }^{\circ} \mathrm{C}-1 \mathrm{~h}$ & 100 & $30 \mathrm{~Hz}$ & 45 \\
\hline $\mathrm{Ni}_{0,5}^{0,3} \mathrm{Zn}_{0,5} \mathrm{Fe}_{2} \mathrm{O}_{4}$ & Síntese por combustão & $1250{ }^{\circ} \mathrm{C}-3 \mathrm{~h}$ & $81^{*}$ & $1 \mathrm{MHz}$ & 46 \\
\hline $\mathrm{Ni}_{0,6}^{0,3} \mathrm{Zn}_{0,4}^{0,3} \mathrm{Fe}_{2}^{2} \mathrm{O}_{4}^{4}$ & Convencional & $1200{ }^{\circ} \mathrm{C}-6 \mathrm{~h}$ & 22,28 & $100 \mathrm{kHz}$ & 44 \\
\hline $\mathrm{Ni}_{0,6}^{0,6} \mathrm{Zn}_{0,4}^{0,4} \mathrm{Fe}_{2}^{2} \mathrm{O}_{4}^{4}$ & Convencional & $1275^{\circ} \mathrm{C}-0,5 \mathrm{~h}$ & 328 & $1 \mathrm{kHz}$ & 35 \\
\hline $\mathrm{Ni}_{0,6}^{0,6} \mathrm{Zn}_{0,4}^{0,4} \mathrm{Fe}_{2}^{2} \mathrm{O}_{4}^{4}$ & Convencional & Microondas $1275^{\circ} \mathrm{C}-0,5 \mathrm{~h}$ & 17,7 & $1 \mathrm{kHz}$ & 35 \\
\hline $\mathrm{Ni}_{0,6}^{0,6} \mathrm{Zn}_{0,4}^{0,4} \mathrm{Fe}_{2}^{2} \mathrm{O}_{4}^{4}$ & Síntese por combustão & $1250{ }^{\circ} \mathrm{C}-3 \mathrm{~h}$ & $79^{*}$ & $1 \mathrm{MHz}$ & 46 \\
\hline $\mathrm{Ni}_{0,65}^{0,6} \mathrm{Zn}_{0,35}^{0,4} \mathrm{Fe}_{2} \mathrm{O}_{4}^{4}$ & Precursor de citrato & $1200{ }^{\circ} \mathrm{C}-1 \mathrm{~h}$ & $150^{*}$ & $30 \mathrm{~Hz}$ & 45 \\
\hline $\mathrm{Ni}_{0,7}^{0,03} \mathrm{Zn}_{0,3}^{0,35} \mathrm{Fe}_{2} \mathrm{O}_{4}^{4}$ & Síntese por combustão & $1250^{\circ} \mathrm{C}-3 \mathrm{~h}$ & $68^{*}$ & $1 \mathrm{MHz}$ & 46 \\
\hline $\mathrm{Ni}_{0,8} \mathrm{Zn}_{0,2} \mathrm{Fe}_{2} \mathrm{O}_{4}$ & Convencional & $1200^{\circ} \mathrm{C}-6 \mathrm{~h}$ & 28,23 & $100 \mathrm{kHz}$ & 44 \\
\hline $\mathrm{Ni}_{0,8}^{0,8} \mathrm{Zn}_{0,2} \mathrm{Fe}_{2} \mathrm{O}_{4}$ & Convencional & $1275^{\circ} \mathrm{C}-0,5 \mathrm{~h}$ & 206 & $1 \mathrm{kHz}$ & 35 \\
\hline $\mathrm{Ni}_{0,8}^{0, \mathrm{O}} \mathrm{Zn}_{0,2} \mathrm{Fe}_{2} \mathrm{O}_{4}$ & Convencional & Microondas $1275^{\circ} \mathrm{C}-0,5 \mathrm{~h}$ & 110 & $1 \mathrm{kHz}$ & 35 \\
\hline $\mathrm{Ni}_{0,8}^{0,8} \mathrm{Zn}_{0,2}^{0,2} \mathrm{Fe}_{2}^{2} \mathrm{O}_{4}^{4}$ & Precursor de citrato & $1200^{\circ} \mathrm{C}-1 \mathrm{~h}$ & $43^{*}$ & $30 \mathrm{~Hz}$ & 45 \\
\hline $\mathrm{Ni}_{0,8}^{0,8} \mathrm{Zn}_{0,2} \mathrm{Fe}_{2} \mathrm{O}_{4}^{4}$ & Síntese por combustão & $1250{ }^{\circ} \mathrm{C}-3 \mathrm{~h}$ & $51^{*}$ & $1 \mathrm{MHz}$ & 46 \\
\hline $\mathrm{Ni}_{0,8}^{0,8} \mathrm{Zn}_{0,2} \mathrm{Fe}_{2} \mathrm{O}_{4}^{4}$ & Síntese por combustão & $1250{ }^{\circ} \mathrm{C}-3 \mathrm{~h}$ & $60 *$ & $10 \mathrm{kHz}$ & 47 \\
\hline
\end{tabular}

*Valores aproximados, retirados de gráficos.

tendência ao aumento de $\varepsilon^{\prime}$ com o aumento do teor de zinco. Já no que diz respeito ao parâmetro tg $\delta$, a posição relativa das curvas, que foram traçadas para cada composição, variou com a freqüência. A tendência observada na maioria das composições foi um aumento de $\operatorname{tg} \delta$ com a freqüência, até um valor a partir do qual o parâmetro começou a decrescer. A freqüência correspondente a esses picos variou de $600 \mathrm{kHz}$ a $900 \mathrm{kHz}$, conforme a composição. No entanto, algumas curvas de $\operatorname{tg} \delta$ em função da freqüência mostradas por outros autores [45], para ferritas $\mathrm{Ni}_{1-\mathrm{x}} \mathrm{Zn}_{\mathrm{x}} \mathrm{Fe}_{2} \mathrm{O}_{4}$ obtidas pelo método do precursor de citrato, apresentaram comportamento diferente deste: em algumas, a tendência observada para $\operatorname{tg} \delta$ foi uma queda brusca com o aumento da freqüência.

Em outro trabalho [43] são mostradas as propriedades dielétricas de ferritas $\mathrm{Ni}_{1-\mathrm{x}} \mathrm{Zn}_{\mathrm{x}} \mathrm{Fe}_{2} \mathrm{O}_{3}$, com x entre 0,2 e 0,5, obtidas pelo método de síntese por combustão. Os valores obtidos para a constante dielétrica a $1 \mathrm{MHz}$ variaram entre 40 e 90, dependendo da composição química e da temperatura de sinterização, sendo o valor máximo obtido para $\mathrm{x}=0,5 \mathrm{a}$ $1350{ }^{\circ} \mathrm{C}$ e o mínimo para $\mathrm{x}=0,2 \mathrm{a} 1150{ }^{\circ} \mathrm{C}$.

Alguns trabalhos relatam resultados de ferritas Ni-Zn, obtidas pelo método cerâmico convencional, com valores de constante dielétrica mais elevados do que os citados anteriormente. Podese citar como exemplos a ferrita $\mathrm{Ni}_{0,8} \mathrm{Zn}_{0,2} \mathrm{Fe}_{2} \mathrm{O}_{4}$ mostrada em (47) com constante dielétrica próxima de $10^{3} \mathrm{a} 10 \mathrm{kHz}$ e a ferrita $\mathrm{Ni}_{0,65} \mathrm{Zn}_{0,35} \mathrm{Fe}_{2} \mathrm{O}_{4}[48,49]$ com constante dielétrica chegando a mais de $10^{6} \mathrm{em}$ freqüências abaixo de $10 \mathrm{kHz}$.

\section{Influência de outros elementos}

A literatura mostra que é prática comum a adição de dopantes às ferritas Ni-Zn com o objetivo de ajustar as suas propriedades.

Os elementos adicionados alterarão o peso específico da ferrita e também poderão alterar o parâmetro de rede, se incorporados à rede cristalina da ferrita. O peso específico é um detalhe a ser levado em consideração quando a ferrita será parte integrante de estruturas aeroespaciais. As interações eletrônicas do íon adicionado com a estrutura cristalina da ferrita são também aspectos importantes na determinação das propriedades elétricas e magnéticas finais da ferrita.

Os íons adicionados à ferrita poderão ser incorporados pela estrutura espinélio, ocupando as posições A ou B. A posição preferencial de alguns cátions na estrutura espinélio foi definida por diversos pesquisadores, com base em dados termodinâmicos [7]: posição preferencial $\mathbf{A}: \mathrm{Cd}^{2+}, \mathrm{Co}^{2+}, \mathrm{Ti}^{4+}$, $\mathrm{Zn}^{2+}$; posição preferencial $\mathbf{B}: \mathrm{Al}^{3+}, \mathrm{Cr}^{3+}, \mathrm{Cu}^{2+}, \mathrm{Mg}^{2+}, \mathrm{Ni}^{2+}$, $\mathrm{V}^{3+}$; indiferente: $\mathrm{Fe}^{3+}, \mathrm{Mn}^{2+}$

Em uma escala mais abrangente, os elementos adicionados poderão influenciar as propriedades das ferritas por meio de alterações microestruturais. Pode-se citar como exemplo dessas alterações: diminuição do tamanho de grão, como conseqüência da precipitação de fases nos contornos de grão; formação de fase líquida nos contornos de grão durante a sinterização, o que facilita a densificação em 
Tabela IV - Efeitos de íons divalentes em uma ferrita $\mathrm{Ni}_{0,5} \mathrm{Zn}_{0,5} \mathrm{Fe}_{2} \mathrm{O}_{4}[40]$.

[Table IV - Effects of divalent ions on a $\mathrm{Ni}_{0.5} \mathrm{Zn}_{0.5} \mathrm{Fe}_{2} \mathrm{O}_{4}$ ferrite [40].]

\begin{tabular}{cccc}
\hline $\begin{array}{c}\text { Íon } \\
\text { substituto }\end{array}$ & $\begin{array}{c}\text { Parâmetro } \\
\text { de rede }(\AA)\end{array}$ & $\begin{array}{c}\text { Massa espec. } \\
\left(\mathrm{g} / \mathrm{cm}^{3}\right)\end{array}$ & $\begin{array}{c}\text { Permeabilidade } \\
\text { magnética }\end{array}$ \\
\hline $\begin{array}{c}\text { Sem } \\
\text { substituição }\end{array}$ & 8,390 & 4,63 & 307 \\
$\mathrm{Mg}^{2+}$ & 8,392 & 4,18 & 396 \\
$\mathrm{Cu}^{2+}$ & 8,420 & 4,85 & 1160 \\
$\mathrm{Co}^{2+}$ & 8,400 & 4,35 & 4,8 \\
$\mathrm{Mn}^{2+}$ & 8,408 & 4,22 & 292 \\
$\mathrm{Cd}^{2+}$ & 8,415 & 4,58 & 522 \\
$\mathrm{Ca}^{2+}$ & 8,435 & 4,35 & 19,1 \\
\hline
\end{tabular}

temperaturas de sinterização mais baixas. A fase precipitada nos contornos de grão normalmente contribui com o aumento da resistividade da ferrita.

A Tabela IV mostra dados da literatura [40] sobre o efeito da substituição de íons divalentes $\left(\mathrm{Me}^{2+}\right)$ nas propriedades magnéticas e elétricas de ferritas do tipo $\mathrm{Ni}_{0,25} \mathrm{Me}_{0,25} \mathrm{Zn}_{0,5} \mathrm{Fe}_{2} \mathrm{O}_{4}$. A ferrita indicada como "sem substituição" corresponde a uma ferrita do tipo $\mathrm{Ni}_{0,5} \mathrm{Zn}_{0,5} \mathrm{Fe}_{2} \mathrm{O}_{4}$. Percebe-se que o parâmetro de rede tende a aumentar com o aumento do tamanho do raio iônico do íon substituto (na tabela, os íons substitutos estão em ordem crescente de raio iônico).

Da Tabela IV observa-se que o cobre foi capaz de aumentar bastante a permeabilidade magnética da ferrita Ni$\mathrm{Zn}$. Este comportamento tem sido confirmado por diversos trabalhos sobre ferritas Ni-Cu-Zn [50, 51]. Por outro lado, é verificado da mesma tabela que o cobalto reduz bastante essa propriedade, sendo este comportamento também confirmado por outros trabalhos da literatura $[52,53]$.

Foi estudado o efeito dos aditivos $\mathrm{CaO}, \mathrm{Na}_{2} \mathrm{O}, \mathrm{ZrO}_{2}, \mathrm{Li}_{2} \mathrm{O}$, $\mathrm{K}_{2} \mathrm{O}$ e $\mathrm{Sb}_{2} \mathrm{O}_{3}$ nas propriedades de uma ferrita $\mathrm{Ni}_{0,36} \mathrm{Zn}_{0,64} \mathrm{Fe}_{2} \mathrm{O}_{3}$ fabricada pelo método cerâmico convencional [54, 55]. Verificou-se que dos aditivos estudados o $\mathrm{Li}_{2} \mathrm{O}$ foi o que promoveu um maior aumento da permeabilidade magnética a $1 \mathrm{kHz}$ e que as combinações $\mathrm{Na}_{2} \mathrm{O}-\mathrm{CaO}-\mathrm{Li}_{2} \mathrm{O}$ e $\mathrm{Na}_{2} \mathrm{O}-\mathrm{CaO}-$ $\mathrm{ZrO}_{2}$ proporcionaram aumento da densidade, chegando a um valor de $98,1 \%$.

A permeabilidade magnética e a constante dielétrica de uma ferrita $\mathrm{Li}_{0,2} \mathrm{Ni}_{0,3} \mathrm{Zn}_{0,3} \mathrm{Fe}_{2,2} \mathrm{O}_{4}$, fabricada pelo método cerâmico convencional, foram estudadas [56], em freqüências de 1 a $13 \mathrm{GHz}$. Verificou-se que a constante dielétrica se mantém constante, em um valor de cerca de 7,5 nessa faixa de freqüência. A permeabilidade magnética apresentou um valor de 3 na freqüência de $1 \mathrm{GHz}$ e chegou próximo de zero a cerca de $5 \mathrm{GHz}$.

A literatura [57] afirma que os aditivos $\mathrm{CaO}, \mathrm{SiO}, \mathrm{TiO}_{2}$, $\mathrm{Nb}_{2} \mathrm{O}_{5}, \mathrm{Ta}_{2} \mathrm{O}_{5}, \mathrm{~V}_{2} \mathrm{O}_{5}$, e $\mathrm{HfO}_{2}$ tendem a aumentar a resistividade das ferritas (agindo nos contornos dos grãos ou no interior dos mesmos), consequentemente diminuindo as perdas

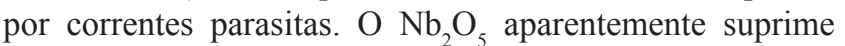
a evaporação do zinco, diminuindo as tensões internas.
$\mathrm{Na}$ fração de aproximadamente 200 ppm, a adição $\mathrm{Nb}_{2} \mathrm{O}_{5}$ resultou em uma microestrutura com grãos praticamente isentos de defeitos nos contornos.

Foi estudado o efeito da adição de $\mathrm{V}_{2} \mathrm{O}_{5}$ nas propriedades de uma ferrita do tipo $\mathrm{Ni}_{0,62} \mathrm{Zn}_{0,40} \mathrm{Fe}_{1,98} \mathrm{O}_{4}$ fabricada pelo método cerâmico convencional e verificou-se que a permeabilidade magnética inicial aumentou com o aumento do teor de $\mathrm{V}_{2} \mathrm{O}_{5}$ até $0,4 \%$ molar, chegando a uma valor pouco acima de $300 \mathrm{a} 1 \mathrm{kHz}$ $[58,59]$. Com um teor acima de $0,4 \%, \mu$ ' caiu abruptamente. $\mathrm{O}$ teor de $0,4 \% \mathrm{~V}_{2} \mathrm{O}_{5}$ molar também foi o correspondente ao menor fator de perdas encontrado no trabalho.

Chandra Prakash e Baijal [60] estudaram o efeito do titânio no comportamento dielétrico entre $100-500 \mathrm{kHz}$ de uma ferrita do tipo $\mathrm{Zn}_{0,25} \mathrm{Ni}_{0,75+\mathrm{t}} \mathrm{Ti}_{\mathrm{t}} \mathrm{Fe}_{2-2 \mathrm{t}} \mathrm{O}_{4}$, com $\mathrm{t}$ variando de 0 a 0,5 . A constante dielétrica e o parâmetro tg $\delta$ a $2 \mathrm{kHz}$ diminuíram com o aumento do teor de titânio, conforme mostra a Tabela V.

Um estudo [61] sobre o efeito da adição de $\mathrm{SiO}_{2}$ e $\mathrm{GeO}_{2}$

Tabela V - Influência do teor " $t$ " de titânio nas propriedades dielétricas a $2 \mathrm{kHz}$ de uma ferrita $\mathrm{Zn}_{0,25} \mathrm{Ni}_{0,75+\mathrm{t}} \mathrm{Ti}_{\mathrm{t}} \mathrm{Fe}_{2-2 \mathrm{t}} \mathrm{O}_{4}$ [60]. [Table V - Influence of the titanium fraction " $t$ " on the dielectric properties of a $\mathrm{Zn}_{0.25} \mathrm{Ni}_{0.75+t} \mathrm{Ti}_{t} \mathrm{Fe}_{2-2 t} \mathrm{O}_{4}$ ferrite at 2 $\mathrm{kHz}$ [60].]

\begin{tabular}{ccccccc}
\hline$t=$ & 0,0 & 0,1 & 0,2 & 0,3 & 0,4 & 0,5 \\
$\varepsilon^{\prime}=$ & 5530 & 1580 & 31,3 & 15,9 & 12,6 & 8,5 \\
$\operatorname{tg} \delta=$ & 3,70 & 2,48 & 0,49 & 0,40 & 0,14 & 0,11 \\
\hline
\end{tabular}

nas propriedades magnéticas de ferritas $\mathrm{Ni}_{0,58} \mathrm{Zn}_{0,40} \mathrm{Fe}_{2,04} \mathrm{O}_{4}$, fabricadas pelo método cerâmico convencional, demonstrou que a sílica se apresentou praticamente insolúvel e se precipitou por toda a matriz da ferrita. $\mathrm{O} \mathrm{GeO}_{2}$, por sua vez, se mostrou insolúvel para teores $\geq 0,32 \%$ molar, precipitando uniformemente nos contornos de grão. A adição de $\mathrm{GeO}_{2}$ aumentou a permeabilidade magnética e diminuiu as perdas magnéticas, enquanto o $\mathrm{SiO}_{2}$ diminuiu a permeabilidade e as perdas.

Alguns trabalhos [62-64] mostram os efeitos do alumínio e do cromo nas ferritas $\mathrm{Ni}-\mathrm{Zn}$ do tipo $\mathrm{Ni}_{0,7} \mathrm{Zn}_{0,3} \mathrm{Al}_{\mathrm{x}} / \mathrm{Cr}_{\mathrm{x}} \mathrm{Fe}_{2-\mathrm{x}} \mathrm{O}_{4}$, fabricadas pelo método cerâmico convencional. A Tabela VI mostra os resultados obtidos para permeabilidade magnética a $1 \mathrm{kHz}$.

Da Tabela VI, percebe-se que a presença tanto do alumínio

Tabela VI - Permeabilidade magnética a $1 \mathrm{kHz}$ de uma ferrita do tipo $\mathrm{Ni}_{0,7} \mathrm{Zn}_{0,3} \mathrm{Me}_{\mathrm{x}} \mathrm{Fe}_{2-\mathrm{x}} \mathrm{O}_{4}$ para $\mathrm{Me}=\mathrm{Al}$ ou $\mathrm{Cr}$ [64].

[Table VI - Magnetic permeability of a $\mathrm{Ni} \mathrm{Zn}_{0.3} \mathrm{Me}_{x} \mathrm{Fe}_{2-x} \mathrm{O}_{4}$ at $1 \mathrm{kHz}$, where $\mathrm{Me}=\mathrm{Al}$ or $\mathrm{Cr}$ [64].]

\begin{tabular}{ccc}
\hline $\boldsymbol{x}$ & $\mu^{\prime}(\mathrm{Me}=\mathrm{Al})$ & $\mu^{\prime}(\mathrm{Me}=\mathrm{Cr})$ \\
\hline 0 & 238 & 238 \\
0,05 & 231 & 232 \\
0,10 & 227 & 220 \\
0,15 & 178 & 201 \\
0,20 & 155 & 184 \\
0,25 & 120 & 140 \\
\hline
\end{tabular}


quanto do cromo reduziu a permeabilidade magnética, sendo que para teores acima de $\mathrm{x}=0,10$ a redução de $\mu$ ' causada pelo alumínio foi levemente mais acentuada que a causada pelo cromo.

Há diversos trabalhos sobre os efeitos de terras-raras nas propriedades das ferritas Ni-Zn [65-73]. Ainfluência da adição de európio, gadolínioeítrioemferritas dotipo $\mathrm{Ni}_{0,5} \mathrm{Zn}_{0,5} \mathrm{Fe}_{1,98} \mathrm{RE}_{0,02} \mathrm{O}_{4}$ (sendo RE o terra-rara adicionado), fabricadas pelo método cerâmico convencional, foi demonstrada [42]. Entre $1 \mathrm{MHz}$ e $1 \mathrm{GHz}$ observou-se que o gadolínio praticamente não alterou os valores de permeabilidade magnética da ferrita, enquanto o európio e o ítrio reduziram tais valores e resultaram em curvas $\mu^{\prime} \times$ freqüência quase coincidentes. A Tabela VII mostra alguns resultados de permeabilidade magnética a $1 \mathrm{MHz}$ retirados desse trabalho.

Em um estudo sobre o efeito da dopagem com samário de

Tabela VII - Permeabilidade magnética a $1 \mathrm{MHz}$ de uma ferrita $\mathrm{Ni}_{0,5} \mathrm{Zn}_{0,5} \mathrm{Fe}_{1,98} \mathrm{RE}_{0,02} \mathrm{O}_{4}$ [42].

[Table VII-Magneticpermeabilityofa $\mathrm{Ni}_{0.5} \mathrm{Zn}_{0.5} \mathrm{Fe}_{1.98} \mathrm{RE}_{0.02} \mathrm{O}_{4}$ ferrite at $1 \mathrm{MHz}$ [42].]

$\begin{array}{ccccc}R E= & \text { Nenhum } & \mathrm{Y} & \mathrm{Eu} & \mathrm{Gd} \\ \mu^{\prime}= & 135 & 75 & 80 & 138\end{array}$

uma ferrita do tipo $\mathrm{Ni}_{0.5} \mathrm{Zn}_{0.5} \mathrm{Fe}_{2-\mathrm{x}} \mathrm{Sm}_{\mathrm{x}} \mathrm{O}_{4}$ obtida pelo método de síntese por combustão [71], foi observado que a precipitação da fase $\mathrm{SmFeO}_{3}$ inibiu o crescimento de grão da ferrita. Também foi observada uma redução da porosidade e do valor da permeabilidade máxima com o aumento do teor de samário.

Em ferritas do tipo $\mathrm{Ni}_{0,65-\mathrm{x}} \mathrm{Zn}_{0,35+\mathrm{x}} \mathrm{Fe}_{2-\mathrm{x}} \mathrm{Sc}_{\mathrm{x}} \mathrm{O}_{4}$, fabricadas pelo método cerâmico convencional, verificou-se [41] que com o aumento do teor de escândio ocorreu um aumento da permeabilidade magnética e uma diminuição do tamanho dos grãos da ferrita, os quais apresentaram grande quantidade de pequenos poros aprisionados nos seus interiores. A 10 $\mathrm{kHz}$, o valor máximo de $\mu^{\prime}$ apresentado no trabalho foi 499, correspondente à ferrita com maior teor de escândio. A Tabela VIII mostra alguns resultados de permeabilidade magnética a $10 \mathrm{kHz}$ obtidos no trabalho.

Em uma ferrita do tipo $\mathrm{Ni}_{0,7} \mathrm{Zn}_{0,3} \mathrm{Er}_{x} \mathrm{Fe}_{2-\mathrm{x}} \mathrm{O}_{4}$, fabricada

Tabela VIII - Permeabilidade magnética ( $\left.\mu^{\prime}\right)$ a $10 \mathrm{kHz}$ de uma ferrita $\mathrm{Ni}_{0,65-\mathrm{x}} \mathrm{Zn}_{0,35+\mathrm{x}} \mathrm{Fe}_{2-\mathrm{x}} \mathrm{Sc}_{\mathrm{x}} \mathrm{O}_{4}$. [41].

[Table VIII-Magnetic permeability ( $\mu^{\prime}$ ) of a Ni $i_{0.65-x} \mathrm{Zn}_{0.35+x} \mathrm{Fe}_{2-x} \mathrm{Sc}_{x} \mathrm{O}_{4}$ ferrite at $10 \mathrm{kHz}$ [41].]

\begin{tabular}{ccccccc}
\hline$x=$ & 0,000 & 0,025 & 0,050 & 0,075 & 0,100 & 0,125 \\
$\mu^{\prime}=$ & 415 & 429 & 442 & 455 & 471 & 499 \\
\hline
\end{tabular}

pelo método cerâmico convencional [72], observou-se uma tendência ao aumento da constante dielétrica e do parâmetro $\operatorname{tg} \delta$ com o aumento do teor de érbio. A Tabela IX mostra alguns dos resultados obtidos no trabalho.
Tabela IX - Valores de $\varepsilon^{\prime}$ e $\operatorname{tg} \delta$ a $1 \mathrm{MHz}$ para uma ferrita $\mathrm{Ni}_{0,7} \mathrm{Zn}_{0,3} \mathrm{Er}_{\mathrm{x}} \mathrm{Fe}_{2-\mathrm{x}} \mathrm{O}_{4}$ [72].

[Table IX- $-\varepsilon$ 'and tg $\delta$ values at $1 \mathrm{MHz}$ for a $\mathrm{Ni}_{0,7} \mathrm{Zn}_{0.3} \mathrm{Er}_{x} \mathrm{Fe}_{2-x} \mathrm{O}_{4}$ ferrite [72].]

\begin{tabular}{ccc}
\hline $\mathrm{x}$ & $\varepsilon^{\prime}$ & $\operatorname{tg} \delta$ \\
\hline 0,2 & 542 & 0,24 \\
0,4 & 614 & 0,36 \\
0,6 & 712 & 0,42 \\
0,8 & 802 & 0,58 \\
1,0 & 882 & 0,62 \\
\hline
\end{tabular}

Em uma ferrita do tipo $\mathrm{Ni}_{1-\mathrm{x}} \mathrm{Zn}_{\mathrm{x}} \mathrm{Fe}_{1,95} \mathrm{La}_{0,05} \mathrm{O}_{4}$, fabricada pelo método cerâmico convencional, observou-se um aumento significativo da constante dielétrica para valores de $\mathrm{x}$ acima de 0,5 . $\mathrm{O}$ valor mais alto a $400 \mathrm{kHz}$ foi de cerca de 700 para $x=0,5$.

A literatura de patentes contem registros de ferritas com base Ni-Zn e uma combinação de dois dopantes para se ajustar as propriedades elétricas e magnéticas. Tem-se como exemplo as patentes de ferritas Ni-Zn dos seguintes tipos: $\mathrm{Ni}_{X} \mathrm{Zn}_{1-\mathrm{X}} \mathrm{Fe}_{2-\mathrm{Y}-}$ ${ }_{\mathrm{Z}} \mathrm{Mn}_{\mathrm{Y}} \mathrm{M}_{\mathrm{Z}} \mathrm{O}_{4}$ com M $=\mathrm{Sn}$ ou Ge (NEC Corporation) [74], sendo $0,55 \leq \mathrm{X} \leq 1,00 ; 0,001 \leq \mathrm{Y} \leq 0,36 ; 0,002 \leq \mathrm{Z} \leq 0,36 ; \mathrm{Ni}_{\mathrm{X}} \mathrm{Zn}_{1}$. ${ }_{\mathrm{X}} \mathrm{Fe}_{2-\mathrm{Y}-\mathrm{Z}} \mathrm{Mn}_{\mathrm{Y}} \mathrm{Zr}_{\mathrm{Z}} \mathrm{O}_{4}$ (NEC Corporation) [75], sendo: $0,55 \leq \mathrm{X} \leq$ 0,$80 ; 0,001 \leq \mathrm{Y} \leq 0,20 ; 0,001 \leq \mathrm{Z} \leq 0,04 ; \mathrm{Ni}_{\mathrm{x}} \mathrm{Zn}_{\mathrm{y}} \mathrm{Cu}_{\mathrm{z}} \mathrm{Co}_{\varepsilon} \mathrm{Fe}_{2 \pm \delta} \mathrm{O}_{4}$ (Thomson-CSF) [76], sendo: $\mathrm{x}+\mathrm{y}+\mathrm{Z}+\varepsilon=1 \pm \delta \operatorname{com} \delta \leq 0,05 ; \varepsilon$ $\geq 0,04$ (preferencialmente menor que 0,1 ); $0,05 \leq \mathrm{Z} \leq 0,35 ; 0$ $\leq \mathrm{y} \leq 0,65$.

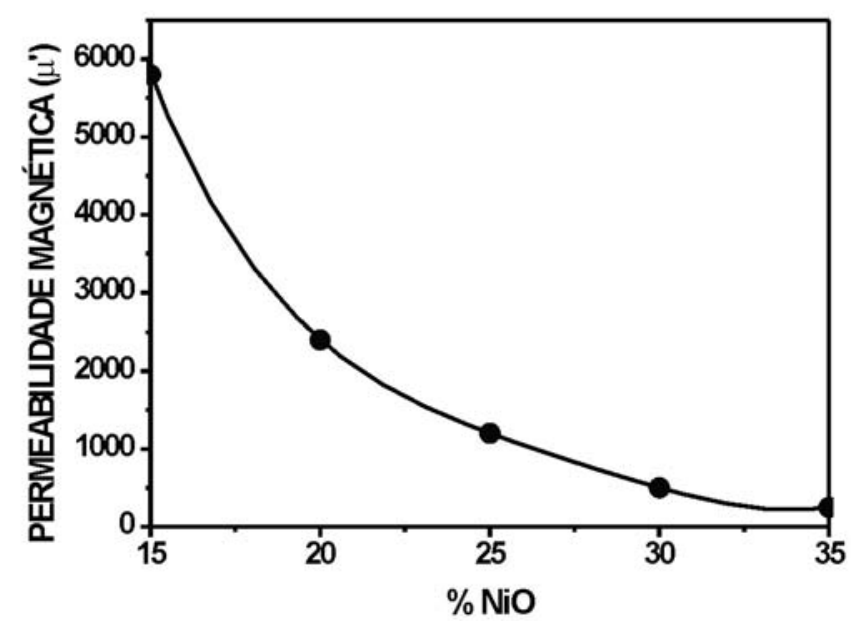

Figura 3: Variação de $\mu$ ', para uma ferrita Ni-Zn fabricada a partir de $50 \% \mathrm{Fe}_{2} \mathrm{O}_{3}$, em função do teor de $\mathrm{NiO}$.

[Figure 3: Relation between $\mu$ 'and the percentage of $\mathrm{NiO}$ for a ferrite fabricated from $50 \% \mathrm{Fe}_{2} \mathrm{O}_{3}$.]

\section{RELAÇÃO ENTRE MICROESTRUTURA E PROPRIEDADES DAS FERRITAS Ni-Zn}

As principais características microestruturais citadas na literatura como sendo fatores que influenciam a 
permeabilidade magnética e a constante dielétrica das ferritas são as relacionadas ao tamanho de grão e à porosidade. No entanto, não é simples distinguir o efeito de cada um desses fatores isoladamente, já que a densificação é acompanhada de crescimento de grão durante a sinterização. Dados sobre a cinética dos processos envolvidos na sinterização de ferritas $\mathrm{Ni}-\mathrm{Zn}$ podem ser encontrados na literatura $[77,78]$.

Alguns modelos para a dependência da constante dielétrica com a porosidade já foram propostos, os quais na maioria das vezes são adaptações de modelos desenvolvidos para materiais compostos, neste caso considerando-se o material um composto formado de sólido + poros. Rice [79] elaborou uma revisão sobre tais modelos, sendo um deles representado na Fig. 4. O modelo, que considera uma concentração diluída de poros esféricos, é definido pela seguinte expressão:

$$
\varepsilon=\varepsilon_{0} \frac{2 \varepsilon_{0}+1+2 P\left(1-\varepsilon_{0}\right)}{2 \varepsilon_{0}+1-P\left(1-\varepsilon_{0}\right)}
$$

Sendo $\mathrm{P}$ a fração volumétrica de poros e $\varepsilon_{0}$ a constante dielétrica do material sem porosidade.

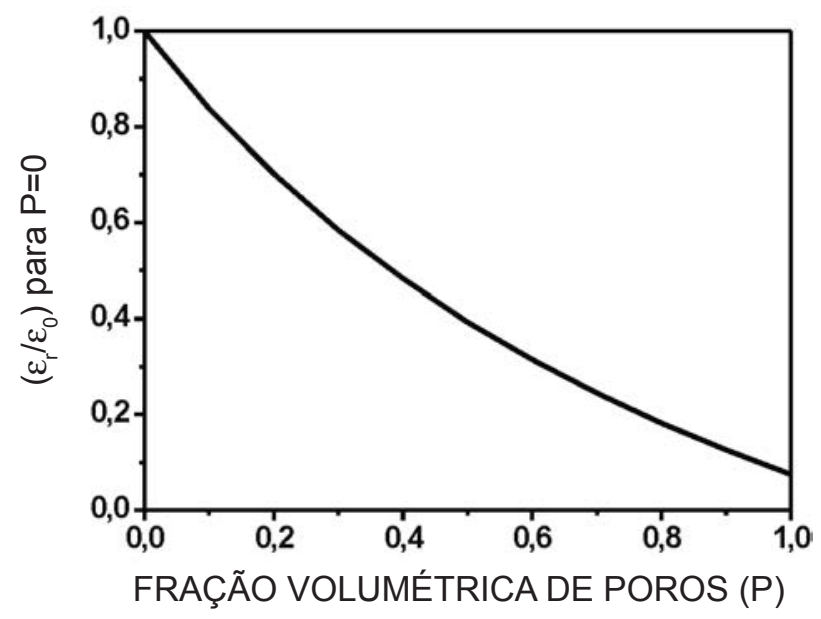

Figura 4: Um dos modelos da literatura, para a variação da constante dielétrica em função da porosidade, citado por Rice [79]. Considerou-se $\varepsilon$ dos poros como 1 e $\varepsilon$ do material como sendo 10 . [Figure 4: A model from literature for variation of dielectric constant with porosity (cited in Rice [79]). It was considered $\varepsilon$ (pores) $=1$ and $\varepsilon$ (material) $=10$.]

Na Fig. 4 percebe-se que a tendência mostrada pelo modelo é uma diminuição da constante dielétrica com o aumento da porosidade. Esta tendência já foi verificada experimentalmente [80] em ferritas Ni-Zn.

A localização dos poros na microestrutura também é um parâmetro a ser controlado nas ferritas. Perduijn e Peloschek [81] explicam que os poros, além de não contribuírem com a magnetização do material, geram campos de desmagnetização quando o material é submetido a um campo magnético alternado. Para se obter uma maior permeabilidade deve-se reduzir a quantidade de poros no interior dos grãos.

A literatura [82] afirma que uma diminuição da taxa de aquecimento durante a sinterização, entre $950{ }^{\circ} \mathrm{C}$ e
$1100{ }^{\circ} \mathrm{C}$, diminuirá a porosidade no interior dos grãos da ferrita. Segundo consta, isso ocorre devido ao retardamento da taxa de crescimento de grão, facilitando a saída do ar aprisionado.

Foipropostoummodelo, baseadoem dadosexperimentais, para a variação da parte imaginária da permeabilidade magnética (perdas magnéticas, $\mu$ ”) em função da porosidade e da microestrutura de ferritas Ni-Zn-Cu [83]. Nesse trabalho, foi constatado que as perdas magnéticas tendem a diminuir com a diminuição da densidade e do tamanho médio de grão e com o aumento da largura de distribuição do tamanho de grão. A expressão proposta pelos autores foi a seguinte:

$$
\mu^{\prime \prime}=1082,2 \varphi_{s} \cdot G_{a} \cdot \exp \left[-\left(\frac{S_{G}}{G_{a}}\right)\right]
$$

Sendo:

$\phi_{\mathrm{s}}=$ densidade relativa após sinterização. A expressão é válida para um valor acima de $80 \%$.

$\mathbf{G}_{\mathrm{a}}=$ Tamanho médio de grão em $\mu \mathrm{m}$

$\mathbf{S}_{\mathrm{G}}{ }_{\mathrm{a}}=$ Largura da distribuição de tamanho de grão (diferença entre o maior e o menor grão da amostra)

Vale ressaltar que as ferritas usadas na definição do

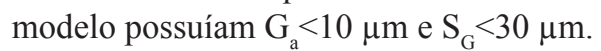

Vários trabalhos tratam da relação entre o tamanho de grão e as propriedades magnéticas das ferritas, dentre os quais os publicados por Globus [84-87] estão entre os precursores. O trabalho de Gieraltowski e Globus [87], no qual foram estudadas ferritas de níquel e granadas, demonstrou que a parte imaginária da permeabilidade magnética ( $\mu$ ”) é proporcional ao tamanho médio de grão $\left(\mathrm{D}_{\mathrm{m}}\right)$. Outro autor [88], estudando uma ferrita do tipo $\mathrm{Ni}_{0,4} \mathrm{Zn}_{0,6} \mathrm{Fe}_{2} \mathrm{O}_{4}$, observou que $\mu$ ' era proporcional a $\left(D_{m}\right)^{1 / 3}$.

Alguns autores estudaram a variação da permeabilidade magnética de ferritas Ni-Zn em função do tamanho de grão e, após ajuste dos valores experimentais obtidos, chegaram às expressões mostradas na Tabela $\mathrm{X}$. No entanto, deve-se observar que a literatura $[4,38,86,87]$ tem mostrado que a relação entre $\mu^{\prime}$ e $D_{m}$ é considerada linear para $D_{m}$ até cerca de $5 \mu \mathrm{m}$, sendo que acima desse valor $\mu$ ' aumenta em taxas menores ou mesmo diminui [88].

Foi verificado [38] que $\mu$ ' aumenta com o aumento de $\mathrm{D}_{\mathrm{m}}$, a uma taxa que se eleva com a diminuição do teor de níquel.

Tabela X - Expressões para a dependência da permeabilidade magnética com o tamanho de grão em ferritas Ni-Zn.

[Table X-Expressions for magnetic permeability dependence on grain size, for Ni-Zn ferrites.]

\begin{tabular}{lcc}
\hline Expressão & \multicolumn{3}{c}{ Composição } & Referência \\
\hline$\mu^{\prime}=39,83+23,47 \mathrm{D}_{\mathrm{m}}(\mathrm{C})$ & $\mathrm{Ni}_{0,7} \mathrm{Zn}_{0,3} \mathrm{Fe}_{2} \mathrm{O}_{4}$ & {$[4]$} \\
$\mu^{\prime}=759,16+972,4 \mathrm{D}_{\mathrm{m}}(\mathrm{D})$ & $\mathrm{Ni}_{0,3} \mathrm{Zn}_{0,7} \mathrm{Fe}_{2} \mathrm{O}_{4}$ & {$[38]$} \\
$\mu^{\prime}{ }_{1 \mathrm{~Hz}}=551,37+166,6 \mathrm{D}_{\mathrm{m}}(\mathrm{E})$ & $\mathrm{Ni}_{0,4} \mathrm{Zn}_{0,6} \mathrm{Fe}_{2} \mathrm{O}_{4}$ & {$[38]$} \\
$\mu^{\prime}{ }_{1 \mathrm{kHz}}=1081+185,8 \mathrm{D}_{\mathrm{m}}(\mathrm{F})$ & $\mathrm{Ni}_{0,35} \mathrm{Zn}_{0,65} \mathrm{Fe}_{2} \mathrm{O}_{4}$ & {$[38]$} \\
\hline
\end{tabular}


Isto provavelmente está relacionado ao fato de ferritas com maior teor de zinco tenderem a apresentar maior tamanho de grão.

\section{CONCLUSÕES}

Estando definidas as metas de propriedades elétricas e magnéticas de uma ferrita Ni-Zn, a literatura contém dados suficientes para auxiliar na seleção da composição química e dos parâmetros de fabricação convencional necessários para o cumprimento destas metas. Novas metodologias, aplicadas tanto na obtenção de ferritas por método químico quanto nas etapas do processo cerâmico convencional, poderão trazer ainda diversas contribuições valiosas na otimização dos processos de fabricação e das propriedades de tais materiais.

Sendo um tipo de material cuja literatura sobre propriedades e processos de fabricação é tão rica, o desenvolvimento de novos dispositivos eletromagnéticos empregando ferritas Ni-Zn, a descoberta de novas aplicações deste material e o desenvolvimento de novos compósitos ficam de certa forma facilitados. Espera-se que os dados e a literatura aqui citados possam auxiliar na fase inicial de investimentos nesse sentido.

\section{REFERÊNCIAS}

[1] Y. C. de Polli, A. C. C. Migliano, C. R. S. Stopa, S. I. Nabeta, J. R. Cardoso, IEEE Trans. Magn. 35, 3 (1999) 1833.

[2] V. L. O. Brito, A. C. C. Migliano, F. C. L. Melo, L. C. Vasconcellos Neto, Anais 49ํㅡㄹ. Cong. Bras. Cerâm., S. Pedro, SP (2005).

[3] T. Tsutaoka, J. Appl. Phys. 93, 5 (2003) 2789.

[4] J. Bera, P. K. Roy, Physica B 363, (2005) 128.

[5] J. L. Snoek, New developments in ferromagnetic materials, Elsevier Publ. Co. Inc., New York, London, Amsterdam, Brussels (1949).

[6] A. Bienkowski, J. Physique 46, 9 (1985) 433.

[7] T. G. Reynolds III, R. C. Buchanan, In: R. C. Buchanan (Ed.): Ceramic Materials for Electronics - Processing, Properties, and Applications, Marcel Dekker Inc., New York (1991) 207.

[8] V. G. Harris, N. C. Koon, C. M. Williams, Q. Zhang, M. Abe, J. P. Kirkland, D. A. McKeown, IEEE Trans. Magn. 31, 6 (1995) 3473.

[9] A. M. El-Sayed, Ceram. Int. 28 (2002) 363.

[10] A. Globus, H. Pascard, V. Cagan, J. Physique, Colloque C1, 38 (1977) 163.

[11] Y.-P. Fu, C.-S. Hsu, K.-W. Tay, Jap. J. Appl. Phys. 44 (2005) 1254.

[12] L. Yu, J. Z. Zhang, Y. Liu, C. Jing, S. Cao, J. Magn. Magn. Mater. 288 (2005) 54.

[13] T. I. Tseng, J. C. Lin, IEEE Trans. Magn. 25, 6 (1989) 4405.

[14] S. Hallynk, G. Pourroy, S. Vilmiont, P.-M. Jacquart, D. Autissier, N. Vukadinovik, H. Pascard, Solid State Sci.
8 (2006) 24.

[15] M. Stefanescu, C. Caizer, M. Stoia, O. Stefanescu, Acta Materialia 54 (2006) 1249.

[16] A. Goldman, in B. B. Ghate, J. J. Simmins (eds.), Magnetic Ceramics: Ceramic Trans., The Am. Ceram. Soc., Westerville, Ohio, USA, 47 (1995) 105.

[17] J. H. Magee, V. Morton, R. D. Fischer, I. J. Lowe, Ferrites: Proc. Int. Conf., Japan (1970) 217.

[18] L. Bruce, in B. B. Ghate, J. J. Simmins (Eds.), Magnetic Ceramics: Ceramic Transactions, The Am. Ceram. Soc., Westerville, Ohio, USA, 47 (1995) 59.

[19] L. Zhiyuan, X. Maoren, Z. Qingqiu. J. Magn. Magn. Mater. 219 (2000) 9.

[20] A. Globus, R. V. Monjaras, IEEE Trans. Magn. 11, 5 (1975) 1300.

[21]A. J. Moulson, J. M. Herbert, Electroceramics: Materials - Properties - Applications, Chapman \& Hall, London (1990) 90.

[22] G. R. Chol, J. Am. Ceram. Soc. 54, 1 (1970) 34.

[23] D. M. Owen, A. T. Chokshi, Y. Ma, T. G. Langdon, in Robert Freer (Ed.), British Ceramic Proceedings - Nanoceramics, The Institute of Materials, London (1993) 61.

[24] M. H. Khedr, A. A. Omar, M. I. Nasr, E. K. Sedeek, J. Anal. Appl. Pyrolysis 76 (2006) 203.

[25] A. Gonchar, S. Gorelik, S. Katynkina, L. Letyuk, I. Ryabov, J. Magn. Magn. Mater. 215-216 (2000) 221.

[26] F. Toussaint, D. Bouvard, P. Tenaud, E. Di Marcello, J. Mater. Proc. Techn. 147 (2004) 72.

[27] P. A. Oberbeck, in B.B. Ghate, J. J. Simmins (eds.): Magnetic Ceramics: Ceram. Trans., The Am. Ceram. Soc., Westerville, Ohio, USA, 47 (1995) 195.

[28] G. P. Dransfield, P. McColgan, I. Birkby, J. R. G. Evans, J. H. Song, in Robert Freer (Ed.), British Ceramic Proceedings - Nanoceramics, The Institute of Materials, London (1993) 23.

[29] C. Heck, Magnetic Materials and their Applications, London Butterworths (1974) 493-497.

[30] C. Heck, J. Weber, Ceramic Industry (November 1961) 74 .

[31] J. M. Blank, J. Appl. Phys. 32, 3 (1961) 378S.

[32] K. Majima, M. Hasegawa, S. Katsuyama, S. Mishima, H. Nagai, Mater. Trans. JIM, 36, 11 (1995) 1392.

[33] K. Majima, M. Hasegawa, M. Yokota, S. Mishima, H. Nagai, J. Japan Inst. Metals 56, 2 (1992) 218.

[34] M. Sorescu, L. Diamandescu, R. Peelamedu, R. Roy, P. Yadoji, J. Magn. Magn. Mater. 279 (2004) 195.

[35] P. Yadoji, R. Peelamedu, D. Agrawal, R. Roy, Mater. Sci. and Eng. B98 (2003) 269.

[36] C.-Y. Tsay, K.-S. Liu, I. N. Lin, J. Eur. Ceram. Soc. 21 (2001) 1937.

[37] S. Yamamoto, S. Horie, N. Tanamachi, H. Kurisu, M. Matsuura, J. Magn. Magn. Mater. 235 (2001) 218.

[38] M. I. Rosales, M. P. Cuaulte, V. M. Castaño, J. Mater. Sci. 33 (1998) 3665.

[39] R. Lebourgeois, C. Le Fur, M. Lebeyrie, M. Paté, J.-P. Ganne, J. Magn. Magn. Mater. 160 (1996) 329.

[40] E. Rezlescu, L. Sachelarie, P. D. Popa, N. Rezlescu, 
IEEE Trans. Magn. 36, 6 (2000) 3962.

[41] B. Parvatheeswara Rao, K. H. Rao, J. Mater. Sci. Lett. 22 (2003) 1607.

[42] G.-L. Sun, J.-B. Li, J.-J. Sun, X.-Z. Yang, J. Magn. Magn. Mater. 281 (2004) 173.

[43] R. V. Mangalaraja, S. Anathakumar, P. Manohar, F. D. Gnanam, Mater. Sci. and Eng. A355 (2003) 320.

[44] G. Ranga Mohan, D. Ravinder, A.V. Ramana Reddy, B. S. Boyanov, Mater. Lett. 40 (1999) 39.

[45] A. Verma, T. C. Goel, R. G. Mendiratta, M. I. Alam, Mater. Sci. and Eng. B60 (1999) 156.

[46] R. V. Mangalaraja, S. Ananthakumar, p. Manohar, F. D. Gnanam, Materials Letters, 57 (2003) 1151.

[47] R.V. Mangalaraja, S. Ananthakumar, P. Manohar, F. D. Gnanam, J. Magn. Magn. Mater. 253 (2002) 64.

[48] B. Parvatheeswara Rao, K. H. Rao, J. Mater. Sci. 32 (1997) 6049.

[49] B. Parvatheeswara Rao, P. S. V. -Subba Rao, K. H. Rao, J. Mater. Sci. Lett. 15 (1996) 781.

[50] A. Gonchar, V. Andreev, L. Letyuk, A. Shishkanov, V. Mairov, J. Magn. Magn. Mater. 254-255 (2003) 544.

[51] H. Zhang, Z. Ma, J. Zhou, Z. Yue, L. Li, Z. Gui, J. Magn. Magn. Mater. 213 (2000) 304.

[52] A. Beitollahi, M. Hoor, J. Mater. Sci.: Materials in Electronics 14 (2003) 477.

[53] Y. Shimada, N. Matsuhita, M. Abe, K. Kondo, T. Chiba, S. Yoshida, J. Magn. Magn. Mater. 278 (2004) 256.

[54] E. Rezlescu, N. Rezlescu, P. D. Popa, Sensors and Actuators A59 (1997) 292.

[55] E. Rezlescu, N. Rezlescu, C. Pasnicu, M. L. Craus, J. Magn. Magn. Mater. 157/158 (1996) 487.

[56] A. N. Yussof. M. H. Abdullah, S. H. Ahmad, S. F. Jusoh, A. A. Mansor, S. A. A. Hamid, J. Appl. Phys. 92, 2 (2002) 876.

[57] D. Stoppels, J. Magn. Magn. Mater. 160 (1996) 323.

[58] G. C. Jain, B. K. Das, R. B. Tripathi, R. Narayan, J. Magn. Magn. Mater. 14, 1 (1979) 80.

[59] G. C. Jain, B. K. Das, R. B. Tripathi, R. Narayan, IEEE Trans. Magn. MAG-18, 2 (1982) 776.

[60] C. Prakash, J. S. Baijal, J. Less-Common Metals, 107 (1985) 51.

[61] B. K. Das, R. B. Tripathi, S. Singh, IEEE Trans. Magn. MAG-23, 5 (1987) 3808.

[62] A. M. Sankpal, S. S. Suryavanshi, S. V. Kakatkar, G. G. Tengshe, R. S. Patil, N. D. Chaudhari, S. R. Sawant, J. Magn. Magn. Mater. 186 (1998) 349.

[63] A. M. Sankpal, S. V. Kakatkar, R. S. Patil, N. D. Chaudhari, R. K. Kamat, S. M. Kabbur, S. S. Suryavanshi, S. R. Sawant, Mater. Chem. ad Phys. 53 (1998) 77.

[64] A. M. Sankpal, S. V. Kakatkar, R. S. Patil, N. D. Chaudhari, S. R. Sawant, S. S. Suryavanshi, J. Mater. Sci.: Materials in Electronics 9 (1998) 173.

[65] L. M. Salah, Physica Stat. Sol. (a) 203, 2 (2006) 271.
[66] N. Rezlescu, E. Rezlescu, J. Physique IV 7, 1 (1997) 225.

[67] N. Rezlescu, E. Rezlescu, C. Pasnicu, M. L. Craus, J. Phys. Condensed Matter 6, 29 (1994) 5707.

[68] N. Rezlescu, E. Rezlescu, Solid State Comm. 88, 2 (1993) 139.

[69] M. A. Ahmed, N. Okasha, L. Salah, J. Magn. Magn. Mater. 264 (2003) 241.

[70] S. E. Jacobo, S. Duhalde, H. R. Bertorello, J. Magn. Magn. Mater. 272-276 (2004) 2253.

[71] A. C. F. M. Costa, M. R. Morelli, R. H. G. A. Kiminami, J. Mater. Sci. 39 (2004) 1773.

[72] K. V. Kumar, A. C. S. Reddy, D. Ravinder, J. Magn. Magn. Mater. 263 (2003) 121.

[73] M. A. Ahmed, E. Ateia, L. M. Salah, A. A. El-Gamal, Mater. Chem. and Phys. 92 (2005) 310.

[74] K. Ishikawa, Y. Sasaki, A. Ochi, Y. Okada, M. Furuya, NEC Corporation, Tokyo, Japan, Pat. US005874020A (1999).

[75] K. Ishikawa, Y. Sasaki, A. Ochi, O. Myohga, Y. Okada, NEC Corporation, Tokyo, Japan, Pat. US005876618A (1999).

[76] R. Lebourgeois, J. Ageron, J. C. Bremaud, G. Peyresoubes, J. P. Joulain, Thomson-CSF, Paris, Pat. US006436307B1 (2002).

[77] A. Dias, N. D. S. Mohallem, R. L. Moreira, Mater. Res. Bull. 33, 3 (1998) 475.

[78] A. Barba, C. Clausell, C. Felíu, M. Monzó, J. Am. Ceram. Soc. 87, 4 (2004) 571.

[79] R. W. Rice, Porosity of Ceramics, Marcel Dekker Inc. (1998) 323.

[80] R. V. Mangalaraja, S. Ananthakumar, P. Manohar, F. D. Gnanam, M. Awano, Mater. Lett. 58 (2004) 1593.

[81] D. J. Perduijn, H. P. Peloschek, Proc. Brit. Ceram. Soc. 10 (1968) 263.

[82] Z. Cohn, J. Daly, C. Parker, in B. B. Ghate, J. J. Simmins (Eds.), Magnetic Ceramics: Ceramic Transactions, The Am. Ceram. Soc., Westerville, Ohio, USA, 47 (1995) 269.

[83] A. Barba, C. Clausell, C. Felíu, M. Monzó, J. Am. Ceram. Soc. 87, 7 (2004)1314.

[84] A. Globus, Influence de la structure granulaire sur la dispersion de la perméabilité des ferrites, Tese, Univ. Paris, France (1963).

[85] A. Globus, P. Duplex, IEEE Trans. Magn. MAG-2, 3 (1966) 441.

[86] A. Globus, P. Duplex, M. Guyiot, IEEE Trans. Magn. (1971) 617.

[87] J. Gieraltowski, A. Globus. IEEE Trans. Magn. MAG13, 5 (1977) 1357.

[88] S. X. Li, IEEE Trans. Magn. MAG-22, 1 (1986) 14.

[89] K. Kondo, T. Chiba, S. Yamada, R. Lebourgeois, J. Ageron, J. C. Bremaud, G. Peyresoubes, J. P. Joulain, 254255 (2003) 541.

(Rec. 14/12/2005, Rev. 02/05/2006, Ac. 11/08/2006) 